\title{
Negative self-beliefs mediate the relationship between depression and suicide ideation among the elderly population: a cross-sectional study
}

\section{Xinfeng Cheng}

Economics and Management Department, Xi'an Technological University

$\mathrm{He} \mathrm{Bu}$

City University of Hong Kong

Wenjie Duan ( $\nabla$ duan.w@outlook.com )

East China University of Science and Technology https://orcid.org/0000-0002-1251-3685

\section{Research article}

Keywords: negative self-beliefs, suicide ideation, depression symptoms, negative self-deprecation, mediate

Posted Date: April 1st, 2020

DOl: https://doi.org/10.21203/rs.3.rs-18833/v1

License: (c) (i) This work is licensed under a Creative Commons Attribution 4.0 International License. Read Full License 


\section{Abstract}

Background High suicide risk among the aged population is a growing concern. Research has linked depression symptoms to suicidal ideation in the general population. The present study examines whether negative self-beliefs mediate the cross-sectional association between depression symptoms and suicide ideation.

Methods A sample of 67 elderly participants from nursing homes, ranging in age from 62 to 91 years ( $M=$ $80.00, S D=6.67$ years; three participants did not indicate the age), completed the Negative Self Belief Inventory, the Geriatric Suicide Ideation Scale, and the 7-item depression subscale of the Depression Anxiety Stress Scale. Results The results show that the associations between depression symptom, negative selfbeliefs and suicide ideation were positive $(r=.44-.52, p<.001)$. Depression symptoms contributed another $13.70 \%$ explained variances. It independently explained another $5.7 \%$ variances beyond other variables to suicide ideation after the entering of negative self-belief $(B=0.126, t=2.183, p<.05)$. The indirect effect of depression symptoms on suicide ideation through negative self-belief was significant (Effect $=0.223,95 \%$ $\mathrm{BCa} \mathrm{Cl}=[0.034,0.468])$.

Conclusion Depression predicts suicide ideation via negative self -beliefs. The current study, incorporated theoretical assumptions and empirical findings in the context of Chinese culture into western prevalent suicide theories. Treatments focusing on restructuring negative self-beliefs are promising to reduce suicide in older adults in China. Future studies should test whether the mechanism in our study can transcend national and cultural boundaries.

\section{Background}

The World Health Organization [1] estimates that about 800,000 suicide deaths occur worldwide every year. In most countries, the rate of suicide for the elderly is much higher than the rate for any other age [2]. For example, the highest suicide rates have historically been among the oldest Americans in the United States [3]. The suicide rate for the elderly aged over 75 years used to be twice or three times higher than the rate for those under age 25 [4]. In 2016, the global population aged 60 years or over accounted for $12.47 \%$ [5], yet the suicide deaths for this age category accounted for $27.35 \%$ of total deaths by suicide [6].

In China, despite the striking decrease in national suicide rates, the elderly suicide rates have been consistently higher than the general suicide rates, and the elderly suicide has become the predominant type of suicide [7-9]. The increasingly salient position of elderly suicide might result from the magnitude of the urban-rural discrepancy. For example, the most substantial decreases in suicide rates are found in old adults in urban areas and younger women in rural areas. Moreover, it has been reported that the number of elderly suicide deaths in rural areas is five to seven times greater than in cities [8,9], while the ratio of rural/urban elder population aged over 60 is less than 3:1[10]. Therefore, suicides among older people living in rural areas contribute most to the suicide rates in China. The high suicide rate among the elderly has aroused broad public and professional concern, and the elderly population deserves more attention in the campaign for suicide reduction in China. These campaigns call for further research to make a confirmation of the risks of suicide to reduce the suicide rate of the aged. 
Among potential risk factors implicated for suicide, depression is closely related to both suicide ideation and attempts. For example, depression is the most typical psychiatric disorders present among suicide completers in Karachi, Pakistan [11]. Suicide and suicide attempts are closely associated with major depressive disorder in Korea [12]. In a sample of rural Australians with depression, 48\% reported lifetime suicide ideation, and 16\% reported a lifetime suicide attempt [13]. In China, high levels of depression symptom has been a principal risk factor for suicide [14]. In terms of older adults, a cross-sectional study, using a final sample of 75 patients $\left(M_{\text {age }}=72.8\right)$ in Gerontopsychiatry Consultation of Central Hospital in Portugal, has shown a significant positive correlation between suicidal ideation and depression [15]. Another study has suggested that the psychological symptoms of depression are significantly associated with suicide ideation in older adults experiencing feelings of perceived burdensomeness [16]. Moreover, due to the close relationship between depression and suicide, many treatments for suicide prevention choose to focus on relieving depressive symptoms. For example a recent 10-year systematic review of suicide prevention strategies indicates that effective pharmacological and psychological treatments of depression are important for suicide prevention [17].

Nevertheless, it is necessary for researchers to move beyond whether depression is associated with suicide, and to examine how depression is associated with suicide among old adults. Recent studies have identified that depression may not be a direct predictor of suicidality [18-20]; instead, other factors, such as family reaction [21] and professional psychological help-seeking [22], may play an influential role in the relationship between depression and suicide. Clinical psychologists working on suicide research also realize that attributing suicide solely to depression is far from enough, and they attempt to highlight the underlying mechanisms behind the whole process of suicide practice. Shneidman has explored five categories of unfulfilled psychological need (e.g., the thwarted love, the ruptured relationships, assaulted self-image, fractured control, and excessive anger) that result in suicide practice [23]. Joiner [24] has shed light on the missing role of perceived burdensomeness and thwarted belongingness. Functional impairment has also been found to be associated with suicide because these factors engender a perception of burdensomeness on others [25]. Some studies capture perceived burdensomeness differently as part of the broader concept of negative self-beliefs. They highlight the role of cognitions related to negative self-beliefs in transferring depression symptoms into suicide ideation. For instance, Mclean, Zang, Zandberg, et al. [26] have demonstrated the negative thoughts about the self (e.g., "I'm incompetent") significantly contributed to the suicidal ideation.

Negative self-belief is that when people dwell on past social "failures", they generate negative images of themselves in the upcoming situation, and make predictions about poor performance and rejection [27]. Negative self-belief can also be interpreted as the negative thoughts, feelings, physical sensations confirming their negative self-image through social interaction [28]. Clark and Well [28] first classify that there are three types of maladaptive self-beliefs including unconditional beliefs about self (UB, e.g., "people think I'm inferior"), conditional beliefs concerning social evaluation (CB, e.g., "if I don't get everything right, I'll be rejected"), and high standards for social performance (HSP, e.g., "I need to be liked by everyone") [29]. Wong and Moulds [27] has develop a the Self-Beliefs Related to Social Anxiety Scale (SBSA) according to the theoretical model proposed by Clark and Well, and they also examine the validity of the scale on nonclinical population proving the stability of the three-factor structure of the concept of self-belief in consistent 
with the theoretical model [30]. Tang and her colleagues translate the SBSA scale into Chinese, modify the scale according to the Chinese cultural context using both quantitative and qualitative methods, and thereby develop the Negative Self-Belief Inventory (NSBI). NSBI remains the same factor structure of SBSA and thus gains its good cross-cultural validity in the Chinese population, which can be used in our research focusing on the elderly suicide in China [29].

Returning to the relationship between negative self-belief and perceived burdensomeness, Joiner [24] has claimed that perceived burdensomeness is an extreme form of negative self-belief. Depressed people are more likely to generate negative self-evaluation and self-image through social interaction, and hence they experience social ineffectiveness, the most extreme form of which is the expression of burdensomeness [24]. Therefore, he articulates that perceive burdensomeness is a more satisfying concept to be included in his suicide model, which makes his model turn out to be a great success in western suicide studies. The suicide model provided by Joiner [24], also known as the Interpersonal Theory of Suicide (ITS) [16], has become a prevalent theory in suicide research to address the mechanism of suicide. According to ITS, perceived burdensomeness (i.e., sense that one is a burden to others in their self-image) and thwarted belongingness (i.e., a greater sense of alienation from others) are, in particular, directly related to the desire to die by suicide [24]. The ITS theory has obtained a wide range of empirical support [for review see 24]. For instance, a five-month longitudinal study showed that the perceived burdensomeness and thwarted belongingness are two intervening variables between depressive symptoms and suicide ideation [16].

Concerning the role of perceived burdensomeness or the role of negative self-belief in suicide research are both the academic attempts to bring cultural and social perspectives into the suicide interpretation. These attempts can be traced back to Durkheim's classical account for altruistic suicide who argues that high social integration makes self-sacrifice for the benefit of the group possible, and suicidal behaviors are embedded in the cultural context [24]. However, the ITS theory emphasizing the role of perceived burdensomeness and thwarted belongingness in the suicide process might only be applicable in western culture. The current paper focuses on the Chinese population and thus, the Chinese cultural context of suicide deserves further discussion in the following paragraph in order to reevaluate whether including negative self-belief is more appropriate for our suicide model designed for the Chinese population.

In terms of the reasons for elderly suicide in China, there are various accounts for it. The rapid social change is the principal reason because it deconstructs the traditional family-centered values, which emphasize heavily on filial piety and family unity [31]. Two characteristics (i.e., shame and harmony) had regulated the Chinese family relationship and the elderly's expected social support from the younger within Asian families [32]. The shame culture in the Asian family put pressure on those who failed to take their filial piety by labeling their behaviors as "shameful". Anthropology studies suggest that the traditional intergenerational relationship in China is the life-long mutual support between the parent's generation and the children's generation, which is different from the western parent-child relationship. Chinese anthropologist Xiaotong [33] conceptualized the eastern intergenerational relationship as a "mutually feedback model", while the western one is captured as the "relaying model" which means the former generation has an obligation to take care of the latter generation until they grow up, but not vice versa. Therefore, in the traditional Chinese 
culture, children have the moral obligation to take care of their elder parents, and parents would keep an eye on their children even if their children have already grown up, especially in rural China.

However, the rapid social change tears down the traditional intergenerational relationships, and creates individualistic offspring who regard filial piety as a burden but take the parents' contribution as granted. Instead, the culture of the market economy nurtures the rational instrumentality way of thinking and the materialistic world view placing the fulfillment of their materialist desire at the center of life [34]. Researches have shown that the deconstruction of traditional family values leads to the diminution of life goal among the family-centered elderly population. Moreover, the individualistic younger generation failing to take the moral obligation leads to desperate older people and thus increase the possibility of having intergenerational family disputes [35].

Moreover, the widening wealth gap contributes to a highly competitive atmosphere among low-income families. Offspring have neither the financial capacity nor the moral motivation to take care of their aging parents. On the contrary, the traditional family values still have an essential position in the aging parents' minds. Parents are still willing to support their adult offspring, which has been taken advantage of by the offspring generation to alleviate their own financial pressure. Therefore, the low socioeconomic status, especially among those rural families, convert the traditional intergenerational support to intergenerational deprivation. Several sociological fieldworks conducted in central China find that new morals have thereby been constructed by the offspring generation, which normalizes suicide among the elderly and creates new moral values suggesting that the elderly should die by suicide if they can no longer contribute to their children $[34,36]$. The new moral order also suggests that being old is itself a stigmatized identity, and adding an economic burden to their children is shameful, irresponsible and not qualified as a parent $[34,36]$. The new moral value devaluing older people leads to desperate and hopeless elderly, which view themselves as a burden to the family and contribute to the negative self-imagination among the elderly [34,36]. Among these fieldwork findings, however, we can classify the moral discourse and narratives encouraging the elderly to die by suicide into the three aspects of negative self-belief.

First, the high cultural expectation of the elderly to exert themselves and support their younger generation engenders high standards for social performance (HSP) through social interaction. For example, the traditional culture rewards parents for supporting and covering part of the expense for their children to get married and build their own house so that they can be decent and praised by society; otherwise, they cannot maintain their decency (mianzi) in the local society. This belief requires them to fulfill a high standard of social performance [34]. Second, stigmatized meaning associated with being old in the new moral order results in their unconditional negative self-belief (UB). For example, in the interviews, the elderly describe themselves as "worthless (meiyong)", "a burden (tuolei)", and they even report superstitious narratives such as "if old people live too long, they would jeopardize (ke) their younger children's health and bring about unfortunate disasters (huiqi) to the family" [34]. Third, because of the extremely high cultural standard, failing to fulfill the cultural requirements would be judged and attached to undesirable labels. The cultural requirement that the elderly should be able to take care of themselves and even always be supportive leads to the conditional belief (CB) that if failing those requirements, they will become unwelcomed [34]. Returning to the item 10 in the NSBI scale ("If I don't get everything right, people won't like me"), the high standard 
cultural requirements are what count as "everything right" in the Chinese social context here. Therefore, the concept of negative self-belief fits the Chinese context of the elderly suicide better than perceived burdensomeness or belongingness suggested by the ITS model according to the narratives collected from the elderly having suicide ideation in China. In conclusion, giving all the theoretical considerations discussed above, we, therefore, inspect the negative self-believe as the mechanism between depressive symptoms and suicide ideation.

In some studies, negative self-beliefs have an impact on several psychological health factors such as anxiety [29], suicide ideation [18], and posttraumatic stress disorder [37]. $\mathrm{Ng}$, Abbott and Hunt [38] conducted a systematic review of 17 evidence-based studies and identified that negatively perceived selfrelated information (e.g., negative self-imagery) is the key cognitive factor that increases social anxiety in both clinical and non-clinical population. Another study suggests that self-related negative cognition has been seen as important in the development and maintenance of posttraumatic stress disorder symptoms after trauma [37]. Furthermore, based on a series of studies, negative self-belief even plays a significant role in suicide ideation. The feeling of worthlessness, one of the conditional beliefs to measure maladaptive selfbeliefs [30], such as "I must be able to convey a favorable impression to everyone" [28], are more strongly associated with lifetime suicide attempt than depression symptoms in individuals with major depressive symptoms [39]. In addition, self-deprecation and negative self-perceptions serve as underlying vulnerabilities to suicide risk and provide a mechanism for understanding why depression might be related to suicide risk among older military personnel and veterans [40]. Other evidence-based studies [41] have demonstrated that shame which can be characterized that "feeling bad about who you are", is significantly associated with suicidal ideation above and beyond the effects of depression. These studies suggest that depression increases the severity of suicide through negative self-belief processes that are more proximally related to suicide than the depression itself.

Although researches have consistently shown strong associations between depression symptoms and suicide ideation, there are not enough studies focusing on the underlying mechanisms of the elderly suicide, especially in the eastern cultural context. The purpose of the current study was to examine the role of negative self-beliefs on the cross-sectional association between depression and suicide ideation among the elderly. Based on previous research, we hypothesize that negative self-beliefs mediate the relationship between depression symptoms and suicide ideation. The significance of this study lies in the reduction of the rate of suicide among the elderly, which can start through the changing of negative self-beliefs. An effective way to reduce suicide among old adults is to truncate the path from depression to suicide by transforming negative self-beliefs to positive perspectives.

\section{Methods}

\section{Participants and procedure}

This study followed a cross-sectional design. Data collection for the present study was conducted from March 2019 to September 2019 in 9 public nursing homes from 9 districts in Chongqing, China. The data were collected one by one accompanied by an MSW student, who assisted the test and answering of 
questions. The package contained 25 items to measure the negative self-belief, depression symptoms, and suicide ideation (details were described in the Measures section).

The calculation of sample size was conducted using Monte Carlo power analysis simulation [42] with a power of 0.8 , a confidence level (\%) of $95 \%$, total number of 5000 replications, and 20000 Monte Carlo Draws. Accordingly, a sample size of 74 at least participants should be recruited in the current study. However, we finally recruited 67 participants. The sample size of 67 can reach a power of 0.78 , which is a sufficient statistical power to detect mediation effects. Inclusion criteria included a) they volunteer to complete questionnaires; $b$ ) they are in good physical and mental health; $c$ ) they have sufficient knowledge for understanding the questionnaire clearly. Exclusion criteria included a) they are cognitively impaired; $b$ ) they are diagnosed to have mental illness. The written informed consent from all subjects was obtained before the completion.

\section{Measures}

Negative Self-Beliefs Inventory (NSBI). NSBI is a newly developed 8-item self-reporting inventory that measures the negative beliefs related to self $[27,29]$. An 11-point Likert scale was adopted for participants to rate on each item from 0 (do not agree at all) to 10 (strongly agree). The mean score of the total inventory was recommended to calculate. High scores reflect the serious negative self-belief. Tang, Duan, Wang, et al. [29] indicated the sound psychometrics of the inventory in one previous study, including clear and solid factor structure, high internal consistency, and good criterion validity. The Cronbach's alpha coefficient of the current older adults is .76.

Geriatric Suicide Ideation Scale - 10 Items (GSIS-10). Suicide ideation was assessed by the 10-item GSIS, which is a commonly used self-report measurement among older adults [43]. Participants were invited to rate the level of agreement of each item on a 5 -point Likert scale ( $1=$ strongly disagree to $5=$ strongly agree). These items described various factors related to suicide ideation, death ideation, and loss of personal and social worth. High mean scores of the total scale indicate high suicidal ideation. In the current sample, the Cronbach's alpha coefficient was .68.

Depression Anxiety Stress Scale. Depression symptoms were measured by the 7-item depression subscale (e.g., "I felt that life was meaningless") of the Depression Anxiety Stress Scale [44]. Participants' feelings in the past week were indicated on a 4-point Likert scale ( $0=$ did not apply to me at all; $3=$ applied to me very much or most of the time). High mean scores of it reflect severe depression symptoms. The whole scale exhibits good internal consistency and solid factor structure in a previous study [45]. The Cronbach's alpha coefficient in the older adults was .68 .

\section{Data Analysis Plan}

The descriptive and correlation analyses were first conducted. Mean scores and standard deviations were calculated. Partial correlations among the negative self-belief, depression symptoms, and suicide ideation were then calculated with gender and age as control variables. It is expected that all these variables were positively associated. Second, the hierarchical regression was performed to clarify the contributions of 
negative self-belief and depression symptoms in understanding suicide ideation among older adults. Suicide ideation was set as the dependent variable in the hierarchical regression. The gender and age were entered in Step 1, followed by depression symptoms in Step 2, and the negative self-belief was entered in Step 3. An additional explanation was expected to obtain after the entering of negative self-belief in the hierarchical regression. Finally, in order to examine the mediation role of negative self-belief between the relationship of depression symptoms and suicide ideation, Model 4 in PROCESS macro was employed [46]. 5,000 bootstrap samples were set to create $95 \%$ bias-corrected and accelerated (BCa) confidence intervals (Cl) to examine the indirect effects. The $95 \%$ confidence interval does not include zero indicated the significances at .05 level. SPSS 24.0 was used to conduct the above-mentioned analyses.

\section{Results}

\section{Descriptive Statistics and Correlation}

A total of 67 participants completed the questionnaires. Participants included in the study aged from 62 to 91 with a mean age of 80.00 years ( $S D=6.67$ years; three participants did not indicate the age). There are 42 females $(62.70 \%)$ and 25 males (37.30\%). Table 1 summarized the mean and standard deviations of depression symptoms, negative self-belief, and suicide ideation. As expected, the associations between these variables were positive $(r=.44-.52, p<.001)$. After the controlling of gender and age, their relationships were also significant and positive $(r=.39-.48, p<.01)$ (Table 1$)$.

[Insert Table 1]

\section{Hierarchical Regression Analysis}

Results of the hierarchical regression analysis was showed in Table 2 with suicide ideation set as the dependent variable. All VIF values were less than 5 , which indicated that the multicollinearity of the variables was not an issue. In Step 1, only gender was a significant factor $(B=0.404, t=2.671, p<.05)$ to explain $10.50 \%$ variance of suicide ideation. In Step 2 , the depression symptoms entered into the equation, which became the only significant predictor $(B=0.549, t=3.289, p<.05)$ to explain the variance of the dependent variable. Depression symptoms contributed another $13.70 \%$ explained variances. Nevertheless, in the third step, after the entering of negative self-belief $(B=0.126, t=2.183, p<.05)$, it independently explained another $5.7 \%$ variances beyond other variables to suicide ideation.

[Insert Table 2]

\section{Mediation Effect Test}

In order to examine the mediation effect of negative self-belief, Model 4 in PROCESS macro was adopted. Figure 1 illustrated the graphic of the mediation effect test. Depression symptoms has a positive effect on suicide ideation in elderly populations, and the direct effect is $0.412(t=2.243, p<.05 ; 95 \% \mathrm{BCa} \mathrm{Cl}=[0.045$, $0.779])$. Moreover, the indirect effect of depression symptoms on suicide ideation through negative selfbelief was significant $($ Effect $=0.223,95 \% \mathrm{BCa} \mathrm{Cl}=[0.034,0.468])$. 
[Insert Figure 1]

\section{Discussion}

The primary goal of the current study was to determine if the negative self-beliefs mediates the relationship between depression symptoms and suicide ideation among the elderly. As would be expected, the results of the current study indicated that depression is interrelated with suicide ideation and the expected mediation effect of negative self-belief has been supported. Overall, negative self-belief was a significant mediator in the relationship between depression and suicide ideation.

According to Baumeister's Escape Theory of suicide and research in western culture [47, 48], the negative self-beliefs and suicide ideation can be explained as consequences of cognitive deconstruction of suicidal individuals who experience strong negative emotions. More specifically, depressed individuals may shift to concreting focus on negative self-beliefs, such as evaluative concerns perfectionism. Evaluative concerns perfectionism (e.g., "If I fail partly, others act as if it's as bad as being a complete failure" [49]) is characterized by a rigid setting of unrealistic personal standards, chronic concerns about expectations imposed by others, overly negative evaluations of one's own performance, and an inability to accept failure $[48,50,51]$. Experiencing negative internal attribution of self-blame and low self-esteem produced by cognitive deconstruction, some suicidal ideation and attempts may arise to escape from the negative selfbelief consequently [51-56].

However, according to the social context in this research, it is the new moral orders constructed by the offspring generation that become the excessively high standard, or in other words, build the "perfectionism" inside the elderly population, and the elderly are being judged accordingly. In addition, why this is "unrealistic" is because for those older people who are unable to take care of themselves or to support their adult children, they still regard the excessively high standard requirements as their responsibility. The new morals discussed above drive the elderly to take the responsibility, which they can never manage to fulfill. Negative self-belief and suicide ideation are thereby generated suggested by their culture rather than developed as a personal kink that results in the "perfectionism". Negative self-belief discussed here has a broader cultural meaning rather than purely a psychological mechanism.

Clinically, the findings can be employed to improve treatment outcomes with suicidal older adults in the Chinese context. It suggests the need for interventions designed to bolster the cognitive coping skills in the short-term and to build a better social environment for the elderly to reduce suicide in the long-term. For suicidal therapies in the short-term, rational emotive behavior therapy can intervene with suicidal individuals [57], which may be beneficial for those who suffer from self-regulatory failure (e.g., "I'm inadequate" [58]) and irrational self-depreciation beliefs to develop unconditional self-acceptance [59]. Evidence also suggests that cognitive behavioral therapy that targets self-deprecatory beliefs (e.g., shame), the type of which pertains to how we judge ourselves [60] contributes to reductions in suicide among the elderly by cognitive restructuring [61].

In line with IPT and our finding, negative self-beliefs (e.g., perceived burdensomeness) can be reduced by engaging in social activities [62], which can be fulfilled in social work practices aiming at empowerment and 
building self-esteem. With regards to the long-term solution to the elderly suicide, interventions should be implemented to change the elderly-negativity cultural morals by not only the therapists or the social workers, but also by the local government in order to ensure the well-being of the aged population. Moreover, in the macro-level practices, the social welfare system needs to improve the living standard of the elderly so that they can have financial support to better take care of themselves rather than depend heavily on their children, which might facilitate the development of the negative narratives towards the elderly within the family. In conclusion, various interventions aiming at holistically reducing the suicide rates among the elderly should be conducted to alter their negative self-belief as revealed in the current study.

The present research has some limitations. The sample was comprised of elderly from nursing homes in China. Therefore, our findings may only be generalized to similar samples (e.g., lonely aged people) and may not generalized to the elderly living with their families. And because we have examined suicide ideation without studying actual death by suicide, the results may not be generalized to actual suicide. Other limitations of the study include the reliance on self-report data to assess suicide ideation, which can be influenced by demand characteristics and thus susceptible to bias [26]. Cross-sectional studies, although able to test a priori causal models [63], yield weaker causal inferences than those associated with longitudinal designs [46] and preclude casual interpretations regarding mediation and the factors that lead to the development or increase of suicide ideation over time. Longitudinal studies with repeated assessments of the elderly are needed to elucidate how negative self-belief mediate the association between depression and suicide ideation.

\section{Conclusion}

Negative self-beliefs have a mediating role in the association between depression symptoms and suicide ideation among the elderly. Our finding is an integration of the empirical works on suicide conducted in China and the above-mentioned theoretical assumption. This research also echoes and contributes to the prevalent IPT theory by investigating empirical findings on the Chinese context. Further investigation focusing on the elderly suicide in China needs to be placed on the research agenda to explain the role of negative self-belief and the suicidal attempt within the Chinese social context. Research in samples of the elderly in other Chinese contexts (e.g., Hong Kong or overseas Chinese immigrant family), as well as in other countries and cultures, is necessary to determine the generalizability of our findings and conclusions more broadly.

\section{Abbreviations}

UB: Unconditional beliefs about self; CB: Concerning social evaluation; HSP: High standards for social performance; SBSA: VIF: Variance Inflation Factor

\section{Declarations}

\section{Ethics approval and consent to participate}


The protocol was approved by The Human Subject Ethics Committees of the Hospital Affiliated to Chongqing Medical University. Written informed consent was obtained from all participants in the study.

\section{Consent for publication}

Not applicable.

\section{Availability of data and materials}

The datasets used and/or analysed during the current study are available from the corresponding author on reasonable request.

\section{Competing interests}

The authors declare that they have no competing interests.

\section{Funding}

This study was supported by East China University of Science and Technology Supporting Funds for Scientific Research, Strengths-Based Interventions in Multiple Contexts (PI: Prof. Wenjie Duan) and Education Department of Shaanxi Province (No.18JK0370, PI: Dr. Xinfeng Cheng). The funders had no role in the design of the study and collection, analysis, and interpretation of data and in writing the manuscript.

\section{Authors' contributions}

All authors designed the study. XC collected the data. HB and WD analysed the data. All authors wrote the manuscript. All authors read and approved the final manuscript. All authors read and approved the final manuscript.

\section{Acknowledgements}

The authors thank all participants for their generous contributions to this study.

\section{References}

1. World Health Organization. Preventing suicide: A global imperative 2014 [cited 2019 Oct 17]. Available from: https://www.who.int/news-room/fact-sheets/detail/suicide.

2. Hawton K, Van HK. Suicide. Lancet. 2009;373:1372-81. 
3. Conwell Y. Suicide later in life. American Journal of Preventive Medicine. 2014;47:S244-S50.

4. Mello Santos C, Bertolote JM, Wang YP. Epidemiology of suicide in Brazil (1980-2000): characterization of age and gender rates of suicide. Revista Brasileira De Psiquiatria. 2005;27:131-4.

5. United Nations. World Population Prospects 20192016 [cited 2019 Oct 17]. Available from: https://population.un.org/wpp/Download/Standard/Interpolated/.

6. World Health Organization. Disease burden and mortality estimates 2016 [cited 2019 Oct 17]. Available from: https://www.who.int/healthinfo/global_burden_disease/estimates/en/.

7. Zhong B, Chiu HFK, Conwell Y. Elderly suicide trends in the context of transforming China, 1987-2014. Scientific Reports. 2016;6:37724.

8. Li X, Xiao Z, Xiao S. Suicide among the elderly in mainland China. Psychogeriatrics. 2009;9:62-6.

9. Li M, Katikireddi SV. Urban-rural inequalities in suicide among elderly people in China: a systematic review and meta-analysis. International Journal for Equity in Health. 2019;18:2.

10. Population Census Office of the State Council. The 2010 population census of the People's Republic of China. 2010.

11. Khan MM, Mahmud S, Karim MS, Zaman M, Prince M. Case-control study of suicide in Karachi, Pakistan. British Journal of Psychiatry. 2008;193:402-5.

12. Hong JJ. Epidemiologic studies on depression and suicide. Journal of the Korean Medical Association. 2012;55:322-8.

13. Handley T, Rich J, Davies K, Lewin T, Kelly B. The Challenges of Predicting Suicidal Thoughts and Behaviours in a Sample of Rural Australians with Depression. International Journal of Environmental Research and Public Health. 2018;15:928.

14. Phillips MR, Yang G, Zhang Y, Wang L, Ji H, Zhou M. Risk factors for suicide in China: a national casecontrol psychological autopsy study. The Lancet. 2002;360:1728-36.

15. Ponte C, Almeida V, Fernandes L. Suicidal ideation, depression and quality of life in the elderly: Study in a gerontopsychiatric consultation. Spanish Journal of Psychology. 2014;17:e14.

16. Guidry ET, Cukrowicz KC. Death ideation in older adults: psychological symptoms of depression, thwarted belongingness, and perceived burdensomeness. Aging Ment Health. 2016;20:823-30.

17. Zalsman G, Hawton K, Wasserman D, van Heeringen K, Arensman E, Sarchiapone M, et al. Suicide prevention strategies revisited: 10-year systematic review. The Lancet Psychiatry. 2016;3:646-59.

18. Campos RC, Gomes M, Holden RR, Piteira M, Rainha A. Does psychache mediate the relationship between general distress and suicide ideation? Death Studies. 2017;41:241-5.

19. Bryan CJ. Cognitive behavioral therapy for preventing suicide attempts: A guide to brief treatments across clinical settings. Proceedings of the Royal Society B Biological Sciences. 2015;273:1715-27.

20. Campos RC, Holden RR, Laranjeira P, Troister T, Oliveira AR, Costa F, et al. Self-report depressive symptoms do not directly predict suicidality in nonclinical individuals: Contributions toward a more psychosocial approach to suicide risk. Death Studies. 2016;40:335-49.

21. Frey LM, Hans JD, Cerel J. Suicide disclosure in suicide attempt survivors: Does family reaction moderate or mediate disclosure's effect on depression? Suicide and life-threatening behavior. 
2016;46:96-105.

22. Wong J, Brownson C, Rutkowski L, Nguyen CP, Becker MS. A mediation model of professional psychological help seeking for suicide ideation among Asian American and white American college students. Archives of Suicide Research. 2014;18:259-73.

23. Shneidman ES. The suicidal mind New York Oxford Univeristy Press; 1996.

24. Joiner TE. Why people die by suicide Cambridge: Harvard University Press; 2005.

25. Conwell Y, Duberstein PR, Hirsch JK, Conner KR, Eberly S, Caine ED. Health status and suicide in the second half of life. International Journal of Geriatric Psychiatry. 2010;25:371-9.

26. Mclean CP, Zang Y, Zandberg L, Bryan CJ, Gay N, Yarvis JS, et al. Predictors of suicidal ideation among active duty military personnel with posttraumatic stress disorder. Journal of Affective Disorders. 2017;208:392-8.

27. Wong QJJ, Moulds ML. Impact of rumination versus distraction on anxiety and maladaptive self-beliefs in socially anxious individuals. Behaviour Research and Therapy. 2009;47:861-7.

28. Clark DM, Wells A. A cognitive model of social phobia. Social phobia: Diagnosis, assessment, and treatment. New York, NY, US: Guilford Press; 1995. p. 69-93.

29. Tang X, Duan W, Wang Y, Guo P. The development of Negative Self-Beliefs Inventory (NSBI): Cultural adaptation and psychometric validation. PeerJ. 2015;3:e1312.

30. Wong QJJ, Moulds ML. Erratum to: A new measure of the maladaptive self-beliefs in social anxiety: Psychometric properties in a non-clinical sample. Journal of Psychopathology and Behavioral Assessment. 2011;33:285-97.

31. Shek DTL, Chan LK. Hong Kong Chinese parents' perceptions of the ideal child. J Psychol. 1999;133:291-302.

32. Pang EC, Jordan-Marsh M, Silverstein M, Cody M. Health-seeking behaviors of elderly chinese americans: Shifts in expectations. The Gerontologist. 2003;43:864-74.

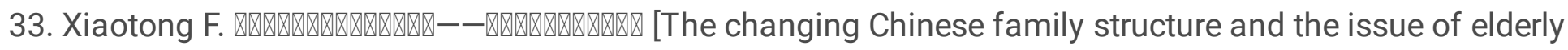
caregiving] Journal of Peking University. 1983:7-16. (Chinese).

34. Chen B. Change of inter-generational relations and the elderly suicide: An empirical stduy in Jingshan county, Hubei province. Sociological Studies. 2009:157-76. (Chinese).

35. Wu F. On "living a life of fortune”. Sociological Studies. 2007:66-85. (Chinese).

36. Yang H, Fan F. Jingshan, Hubei province]. Open Times. 2009:104-25. (Chinese).

37. Cieslak R, Benight CC, Lehman VC. Coping self-efficacy mediates the effects of negative cognitions on posttraumatic distress. Behaviour Research and Therapy. 2008;46:788-98.

38. Ng AS, Abbott MJ, Hunt C. The effect of self-imagery on symptoms and processes in social anxiety: A systematic review. Clinical Psychology Review. 2014;34:620-33.

39. Jeon HJ, Park JI, Fava M, Mischoulon D, Sohn JH, Seong S, et al. Feelings of worthlessness, traumatic experience, and their comorbidity in relation to lifetime suicide attempt in community adults with major depressive disorder. Journal of Affective Disorders. 2014;166:206-12. 
40. Bryan CJ, Roberge E, Bryan AO, Ray-Sannerud B, Morrow CE, Etienne N. Guilt as a mediator of the relationship between depression and posttraumatic stress with suicide ideation in two samples of military personnel and veterans. International Journal of Cognitive Therapy. 2015;8:143-55.

41. Bryan CJ, Morrow CE, Etienne N, Raysannerud B. Guilt, shame, and suicidal ideation in a military outpatient clinical sample. Depression and Anxiety. 2013;30:55-60.

42. Schoemann AM, Boulton AJ, Short SD. Determining Power and Sample Size for Simple and Complex Mediation Models. Social Psychological and Personality Science. 2017;8:379-86.

43. Heisel MJ, Flett GL. The development and initial validation of the geriatric suicide ideation scale. he American Journal of Geriatric Psychiatry. 2006;14:742-51.

44. Lovibond SH, Lovibond PF. Manual for the Depression, Anxiety and Stress Scales (2 ed.). Sydney, Australia: Psychological Foundation; 1995.

45. Wang K, Shi HS, Geng FL, Zou LQ, Tan SP, Wang Y, et al. Cross-cultural validation of the Depression Anxiety Stress Scale-21 in China. Psychological Assessment. 2016;28:E88-E100.

46. Hayes AF. Introduction to mediation, moderation, and conditional process analysis: A regression-based approach. New York: The Guilford Press; 2013.

47. Dean PJ, Range LM. Testing the escape theory of suicide in an outpatient clinical population. Cognitive Therapy and Research. 1999;23:561-72.

48. Blankstein KR, Dunkley DM. Evaluative concerns, self-critical, and personal standards perfectionism: $A$ structural equation modeling strategy. In: Hewitt PL, Flett GL, editors. Perfectionism: Theory, research, and treatment. Washington, DC: American Psychological Association; 2002. p. 285-315.

49. Wheeler HA, Blankstein KR, Antony MM, McCabe RE, Bieling PJ. Perfectionism in anxiety and depression: Comparisons across disorders, relations with symptom severity, and role of comorbidity. International Journal of Cognitive Therapy. 2011;4:66-91.

50. Frost RO, Marten P, Lahart C, Rosenblate R. The dimensions of perfectionism. Cognitive Therapy and Research. 1990;14:449-68.

51. Blankstein KR, Lumley $\mathrm{CH}$, Crawford A. Perfectionism, hopelessness, and suicide ideation: Revisions to diathesis-stress and specific vulnerability models. Journal of Rational-Emotive \& Cognitive-Behavior Therapy. 2007;25:279-319.

52. Stanley IH, Hom MA, Rogers ML, Hagan CR, Joiner TE. Understanding suicide among older adults: A review of psychological and sociological theories of suicide. Aging and Mental Health. 2016;20:113-22.

53. O'Connor RC. The relations between perfectionism and suicidality: A systematic review. Suicide and Life-threatening Behavior. 2007;37:698-714.

54. Chatard A, Selimbegović L. When self-destructive thoughts flash through the mind: Failure to meet standards affects the accessibility of suicide-related thoughts. Journal of Personality and Social Psychology. 2011;100:587-605.

55. Hewitt PL, Flett GL. Perfectionism in the self and social contexts: conceptualization, assessment, and association with psychopathology. Journal of Personality and Social Psychology. 1991;60:456-70.

56. Baumeister R. Suicide as escape from self. Psychological review. 1990;97:90-113. 
57. Ellis A, Ellis TE. Suicide from the perspective of rational emotive behavior therapy. In: Ellis TE, editor. Cognition and suicide: Theory, research, and therapy. Washington, DC, US: American Psychological Association; 2006. p. 75-90.

58. Pychyl TA, Flett GL. Procrastination and self-regulatory failure: An introduction to the special issue. Journal of Rational-Emotive and Cognitive-Behavior Therapy. 2012;30:203-12.

59. Cunningham R, Turner MJ. Using Rational Emotive Behavior Therapy (REBT) with mixed martial arts $(\mathrm{mma})$ athletes to reduce irrational beliefs and increase unconditional self-acceptance. Journal of Rational-Emotive and Cognitive-Behavior Therapy. 2016;34:289-309.

60. Hedman E, Strom P, Stunkel A, Mortberg E. Shame and guilt in social anxiety disorder: Effects of cognitive behavior therapy and association with social anxiety and depressive symptoms. PLoS ONE. 2013;8:e61713.

61. Bhar SS, Brown GK. Treatment of depression and suicide in older adults. Cognitive and Behavioral Practice. 2012;19:116-25.

62. Joiner TE, Van Orde KA, Witte TK, Selby EA, Ribeiro JD, Lewis R, et al. Main predictions of the interpersonal-psychological theory of suicidal behavior: empirical tests in two samples of young adults. Journal of Abnormal Psychology. 2009;118:634-46.

63. Troister T, Holden RR. Factorial differentiation among depression, hopelessness, and psychache in statistically predicting suicidality. Measurement and Evaluation in Counseling and Development. 2013;46:50-63.

\section{Tables}

Table 1

Descriptive Statistics of and Correlations between Variables among the Elderly Population

\begin{tabular}{lccc}
\hline & \multicolumn{3}{c}{ Pearson Correlation (Partial Correlation) } \\
& 1 & 2 & 3 \\
\hline 1 Depression & - & $.52^{* * *}\left(.48^{* * *}\right)$ & $.44^{* * *}\left(.39^{* *}\right)$ \\
2 Negative Self-belief & - & - & $.48^{* * *}\left(.41^{* *}\right)$ \\
3 Suicide Ideation & - & - & - \\
Mean & 0.39 & 1.81 & 2.16 \\
\multicolumn{1}{c}{$S D$} & 0.42 & 1.59 & 0.60 \\
\hline
\end{tabular}

Note. ${ }^{* *} p<.01 ; * * *<<.001$ 
Hierarchical Regression on the Suicide Ideation among the Elderly Population

\begin{tabular}{|c|c|c|c|c|c|c|c|c|c|}
\hline \multirow[t]{3}{*}{ Independent Variables } & \multicolumn{9}{|c|}{ Dependent Variable: Suicide Ideation } \\
\hline & \multicolumn{3}{|c|}{ Step 1} & \multicolumn{3}{|c|}{ Step 2} & \multicolumn{3}{|c|}{ Step3 } \\
\hline & Beta & $t$ & VIF & Beta & $t$ & VIF & Beta & $t$ & VIF \\
\hline Gender & 0.404 & $2.671^{*}$ & 1.003 & 0.287 & 1.980 & 1.068 & 0.193 & 1.311 & 1.168 \\
\hline Age & -0.001 & -0.128 & 1.003 & $<.001$ & -0.008 & 1.005 & $<.001$ & -0.010 & 1.005 \\
\hline Depression Symptoms & & & & 0.549 & $3.289 * *$ & 1.065 & 0.356 & 1.932 & 1.382 \\
\hline Negative Self-belief & & & & & & & 0.126 & $2.183^{*}$ & 1.505 \\
\hline$R^{2}$ & .105 & & & .241 & & & .298 & & \\
\hline$F$ & $3.568 *$ & & & $6.367 * *$ & & & $6.265^{* *}$ & & \\
\hline$\Delta R^{2}$ & & & & & .137 & & .05 & & \\
\hline$\Delta F$ & & & & & 10.8 & $16^{* *}$ & & $64^{*}$ & \\
\hline
\end{tabular}

Note. ${ }^{*} p<.05 ; * * p<.01 ; * * * p<.001$

\section{Figures}

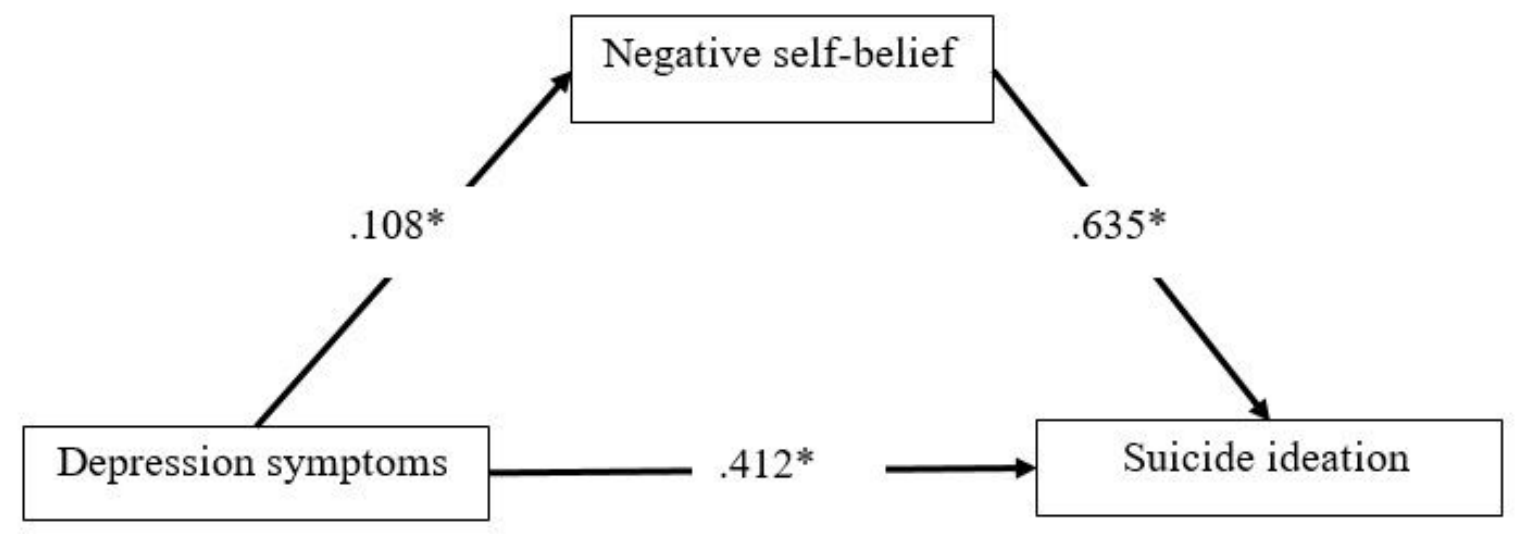

\section{Figure 1}

PROCESS Results for Mediation Model Note. Unstandardized coefficients are presented. * $\mathrm{p}<.05$ 


\section{Supplementary Files}

This is a list of supplementary files associated with this preprint. Click to download.

- STROBEchecklistcrosssectional.doc 